\title{
Local magnetic measurements in magnetic circuits with highly non uniform electromagnetic fields
}

\author{
Ahmed Abou-Elyazied Abdallh, and Luc Dupré \\ Department of Electrical Energy, Systems \& Automation, Ghent University, Ghent, Belgium
}

In this paper, local magnetic measurements are carried out in magnetic circuits with highly non uniform electromagnetic field patterns, including excitation winding and/or air gaps, as in the case of rotating electrical machines. The effect of sensor choice, sensor noise sensitivity, electromagnetic field nonlinearity, and magnetic shielding are investigated. Moreover, the validity of the local magnetic measurements is confirmed by numerical models.

This paper presents a comparison between different sensors to measure local magnetic quantities of two different geometries, shown in Fig. 1a, including advantages, disadvantages, and limitations of each sensor. The local magnetic field strength ' $H_{\text {local }}$ ' is measured using different sensors, such as: flat H-coil, Rogowski coil, Hall-effect, and fluxgate sensors. The local magnetic induction ' $B_{l o c a l}$ ' is measured using the needle probe and the search coil methods [1].

Fig. 11 shows the two studied geometries, made from the same material. The excitation winding is wound over different excitation angles $(\theta)\left[65^{\circ}, 130^{\circ}\right.$, and $\left.230^{\circ}\right]$ of both geometries, to study the effect of field nonlinearity. The introduced air gap of $G_{2}$ is fixed at $1 \mathrm{~mm}$. The quasi static magnetic measurements are performed at $1 \mathrm{~Hz}$ for a sinusoidal current excitation. Fig. 1b shows the measured and simulated $H_{\text {local }}$ at positions 1,2 , and 3, using the flat $\mathrm{H}$-coil, versus different values of the excitation current, in $G_{1}$. It can be observed that the $H_{\text {local }}$ follows Ampere's law at low values of the excitation current (the magnetic permeability ' $\mu$ ' is high, the magnetic flux is approximately confined to the magnetic ring core). At high values of the excitation current, $H_{\text {local }}$ is much less than the values obtained from Ampere's law, where ' $\mu$ ' is dropped gradually and the stray magnetic field is much higher. Moreover, a good correspondence between the simulated and the measured values is observed. In conclusion, Rogowski coils and Hall-effect probes are highly affected by the presence of stray fields, however, flat H-coils and fluxgate sensors are not affected. Fluxgate sensors, with relatively small detectable field range $(0-800 \mathrm{~A} / \mathrm{m})$, are more sensitive than flat $\mathrm{H}$-coils, with unlimited detectable field range.
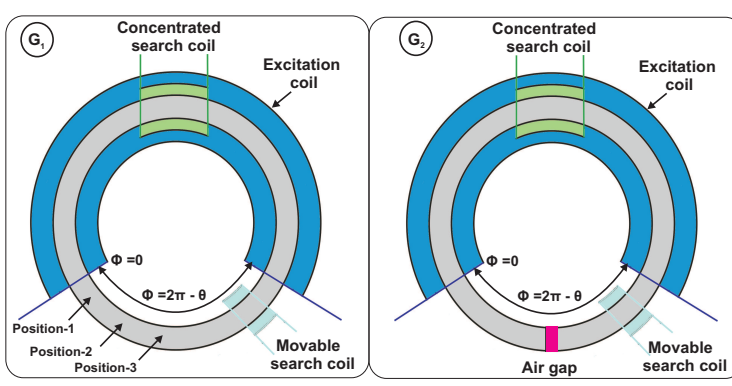

(a)

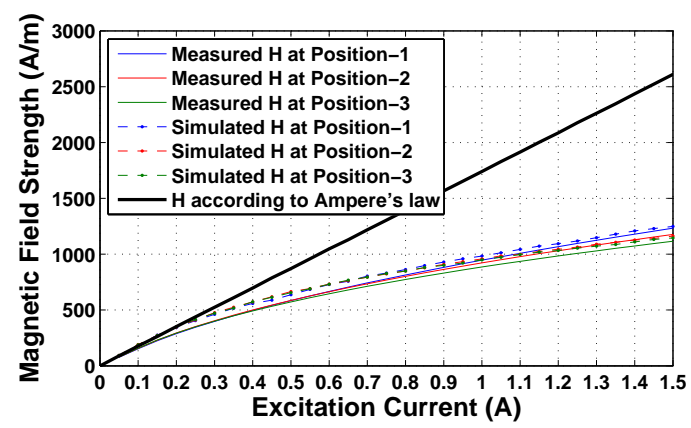

(b)

Figure 1: (a) The two studied geometries. (b)The measured and simulated local magnetic field strength at different positions versus the excitation current, in $G_{1}, \theta=230^{\circ}$.

[1] S. Tumanski, Measured sience \& technology 18-3, (2007), R31-R46. 